\title{
Research on Winding System of Twisting Machine Based on Multi- parameters
}

\author{
Zhang Zhiming , Sun Jun, Wu Xiaoqing \\ School of Electrical \& Mechanical Engineering, Wuhan Textile University, 430073 Wuhan City, China
}

\begin{abstract}
Winding movement of twisting machine is closely related to the package build, package density and yarn quality. The requirements are that the package build is good shaped and the winding tension is proper technically. Namely the angle of winding is fine and there is no overlapping and convex, hard edge on the package. The winding density is same and reasonable on the whole package build. And the fluctuation of winding tension is small. It is becoming development trend that driving of multiple-motors is used on twisting machine. There are many parameters to be controlled such as degree of twist, direction of twist, winding angle and spindle speed and so on. Therefore it is essential to set up reasonable mathematical model of yarn for winding movement.
\end{abstract}

\section{Introduction}

Twisting machine mostly adopted the single motor driving system home and abroad during the past years[1]. Degree of twist is gained by changing the transmission ratio of twist gears. Direction of twist is changed by the rotation direction of the main motor. This can gain the $\mathrm{S}$ twist and $\mathrm{Z}$ twist. The winding movement and the reciprocating yarn guiding movement are realized by the gear wheel and the stepped cam transmission. In this way, twisting movement of the spindle, winding movement of rolls and reciprocating yarn guiding movement are realized on twisting machine[3]. This transmitting is long and the structure is complex. Nowadays, with the development of mechanical and electrical integration technology and demand of saving energy and reducing consumption, multi-motor driving has become the development trend of the multi twisting machine[3-5]. According to the characteristics of traditional twisting machine driving system, this paper puts forward a new twisting machine winding based on multi-parameter controlling system, which can satisfy the whole technological requirements of twisting machine.

\section{Mathematical model of yarn winding movement}

There are several forms such as cylindrical, conical and conical at both ends for the yarn package build. But the principle of these winding movements is same from kinematic of machinery[6].

The yarn winding movement on the twisting machine is the same as the cheese winding machine, spinning frame and roving frame, which are made up of the reciprocating yarn guiding motion and winding rotary movement[7]. In this paper, taking the winding conical package build as an example, the movement of the conical winding package build is shown in Fig. 1.

According to the winding theory of twisting machine, the package build is rotated through friction by the winding roller. According to the basic theory of yarn winding, the relationship between the moving speed $V_{T}$ of winding point $T$ and speed $V_{H}$ of yarn guiding point $\mathrm{H}$ is shown as equation (1).

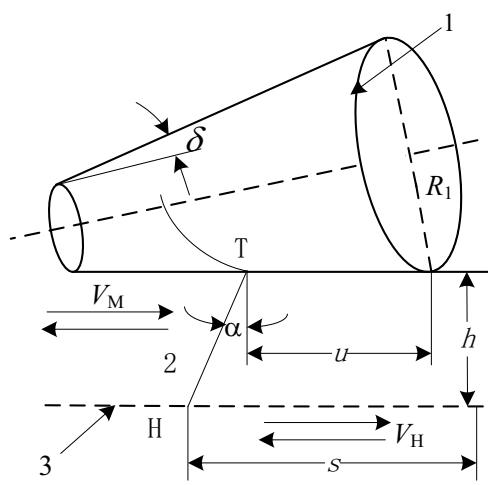

1- package build 2-yarn 3-guiding rod

Figure 1. winding movement of yarn

$$
\begin{gathered}
\frac{d s}{d t}=\frac{d u}{d t}+\frac{h}{\omega\left(R_{1}+u \sin \delta\right)}\left[\frac{d^{2} u}{d t^{2}}-\frac{\sin \delta}{R_{1}-(l-u) \sin \delta}\left(\frac{d u}{d t}\right)^{2}\right] \\
V_{\mathrm{H}}=d s / d t \\
V_{\mathrm{T}}=d u / d t
\end{gathered}
$$


Where $u$ is displacement of winding point $(\mathrm{m}), s$ is distance of guiding rod $(\mathrm{m}), h$ is normal distance of winding point to guiding rod $(\mathrm{m}), \omega$ is rotational angular velocity of the package build ( $\mathrm{rad} / \mathrm{s}), R_{l}$ is big radius of cone package build $(\mathrm{m}), l$ is the length of cone package build(m), $\delta$ is half cone angle of conical package (rad), $t$ is winding time (s).

The winding movement curve is shown as Fig. 2.

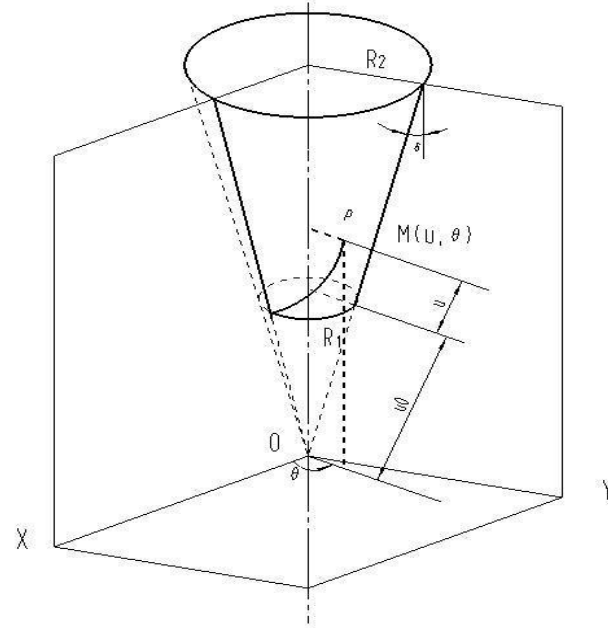

Figure 2. winding movement curve

\section{Mathematical model of yarn guiding movement}

When the winding roller is rotated, the circumferential speed of the points along the bus bar the surface of the roller is not the same at all. It is difficult to achieve the same tension and same density package build at the same time. Intensive analyses show that the tension fluctuation and the density fluctuation are small. And the shape is good by using the appropriate variable acceleration through yarn guiding rule. The practice shows that when the cone angle is small and the speed of guiding rod is not very large. The good winding can also be got with constant velocity yarn guiding rule[8].

At present, with the development and wide application of mechanical and electrical integration technology, the development trend of the high speed and precision winding drive technology is to directly drive guiding rod and yarn guiding parts to realize yarn guiding movement rule by controlling the motor through software. The main advantages are that it abandons the complex cam box and the machine has the advantages of simple structure. It is also very convenient to replace the twist and roll form and very easy to realize high precision winding.

The curve of yarn guiding movement is improved after the transition curve at re-entry point. In Fig 3, the $A B$ and $C D$ segments are the transition curve of the reentry point. $B C$ segment is the middle part of the movement curve. The sine acceleration of the law is used in the vicinity of the two direction-changing points. It uses law of constant velocity to transit in the middle. The reciprocating yarn curve, velocity curve and acceleration curve are shown in Fig.3.

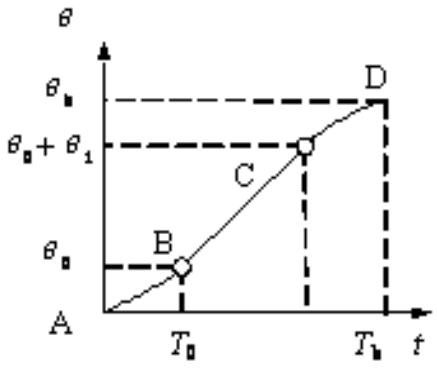

(a) 角位移曲线

$\omega$

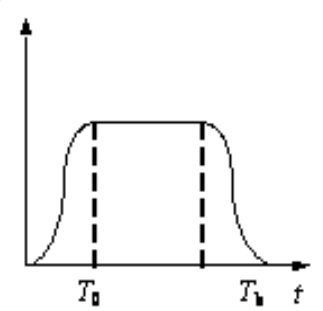

(b) 角速度曲线

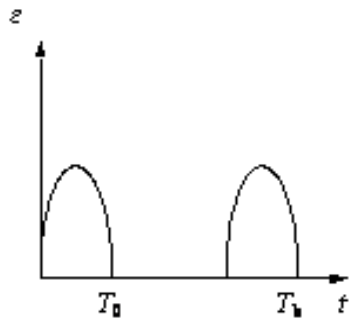

(c) 角加速度曲线

Figure 3. curve of improved yarn guiding movement

The angular displacement of the package build is $\theta$ ( $\mathrm{rad})$, the displacement of the guiding rod is $S(\mathrm{~m})$, then

$$
S=\frac{\theta}{2 \pi} L
$$

Where $L$ is the lead of guiding rod (m).

Therefore, the vertical coordinate of the curve is respectively angular displacement $(\theta)$, angular velocity $(\omega)$, angular acceleration $(\varepsilon)$ of the guiding rod. The horizontal coordinate is the time of movement.

The guiding rod comes and goes both once in a cycle yarn guiding movement. The law of come-and-go movement is basically same, but in the opposite direction. Guiding rod moves forward and reverse correspondingly according to the same curve. Therefore, the movement curve can be studied only in half of the yarn guiding cycle.

From Fig.3, in the travel of yarn guiding movement, the velocity and acceleration at the two re-entry points are zero. The speed of guiding rod reduced to zero and then the guiding rod returned. It can avoid the inertial impact at re-entry point. The maximum acceleration in the whole trip can also be suppressed.

In Fig. 3, the time interval for variable motion near the two re-entry points is $T_{0}$. Displacement is $\theta_{0}$. Half cycle 
is $T_{b}$. The maximum angular displacement is $\theta_{b}$. Then $A B$, $B C, C D$ segment displacement, velocity and acceleration equations are shown as (3)-(11). $A B$ segment: $0 \leq t \leq T_{0}$

$$
\begin{gathered}
\theta_{J}=\theta_{0}\left(\frac{t}{T_{0}}-\frac{1}{\pi} \sin \frac{\pi}{T_{0}} t\right) \\
\omega_{J}=\frac{\theta_{0}}{T_{0}}\left(1-\cos \frac{\pi}{T_{0}} t\right) \\
\varepsilon_{J}=\frac{\pi \theta_{0}}{T_{0}^{2}} \sin \frac{\pi}{T_{0}} t
\end{gathered}
$$

$B C$ segment: $\quad T_{0} \leq t \leq T_{b}-T_{0}$

$$
\begin{gathered}
\theta_{J}=\theta_{0}+\frac{\theta_{1}\left(t-T_{0}\right)}{T_{b}-2 T_{0}} \\
\omega_{J}=\frac{\theta_{1}}{T_{b}-2 T_{0}} \\
\varepsilon_{J}=0
\end{gathered}
$$

$C D$ segment: $T_{b}-T_{0} \leq t \leq T_{b}$

$$
\begin{gathered}
\theta_{J}=-\theta_{0}\left[\frac{T_{b}-t}{T_{0}}-\frac{1}{\pi} \sin \frac{\pi}{T_{0}}\left(T_{b}-t\right)\right]+\theta_{b} \\
\omega_{J}=\frac{\theta_{0}}{T_{0}}\left[1-\cos \frac{\pi}{T_{0}}\left(T_{b}-t\right)\right] \\
\varepsilon_{J}=-\frac{\pi \theta_{0}}{T_{0}^{2}} \sin \frac{\pi}{T_{0}}\left(T_{b}-t\right)
\end{gathered}
$$

where $\theta 、 \omega$ 、 and $\varepsilon$ with the subscript " ${ }_{J}$ " is the basic yarn guiding rule.

There should be proper relation in order to ensure the continuous speed curve.

$$
\frac{\theta_{1}}{\theta_{0}}=\frac{2 T_{b}}{T_{0}}-4
$$

Or:

$$
\frac{T_{b}}{T_{0}}=\frac{\theta_{1}}{2 \theta_{2}}+2
$$

Where $T_{0} 、 T_{1} 、 \theta_{1} 、 \theta_{0}$ are determined by different process conditions and requirements. For example, when $\frac{\theta_{1}}{\theta_{0}}=4, \frac{T_{b}}{T_{0}}=4$, then $\theta_{b}=\theta_{1}+2 \theta_{0}=6 \theta_{0}$,when $\frac{T_{b}}{T_{0}}=6, \frac{\theta_{1}}{\theta_{0}}=8$, then $\theta_{b}=\theta_{1}+2 \theta_{0}=10 \theta_{0}$.

\section{Improvement and anti-aliasing of yarn coiler motion}

\section{1 winding tension improvement}

From the previous analysis, it is known that it is easy to implement equal tension and equal density when winding cylindrical roll, but not for winding cone roll. When winding, line speed of small end is small (less than line speed of the friction roller) and line speed of big end is big. In order to make the winding tension uniform, the winding speed should be as stable as possible. Therefore, in the small end of the yarn bobbin, the guide speed should be larger, in the big end of the yarn bobbin, guide speed should be smaller. There are usually two ways, one is the yarn guiding speed gradually decreases when the yarn is from the small end to big end and it gradually increases when the yarn is from the big end to small end. Another way is to make the time of the reciprocating yarn guiding movement stroke different. The time of big end is a little bigger than the time of small end. In this yarn winding movement, the winding density of small end of the bobbin will be slightly less than that of the big end, but it does not affect the handling and unwinding, so it can still be used.

\section{2 ribbon breaking mechanism of yarn}

There are the following measures to realize ribbon breaking mechanism[9-10]. One way is changing the speed of friction roller periodically to make the roller fast and slow down intermittently. Under the inertial force, the change of bobbin speed always lags behind the change of the friction roller speed, it makes the some regularity transmission relationship between the two is destroyed. So the circle number of yarn winding is not a continuous integer, making it can avoid overlapping.

Another way is changing the yarn guiding speed periodically, making the yarn winding speed change periodically. That also can make the yarn winding cycle not consecutive integer.

In addition, due to the impact of free yarn, the hardness of the bobbin at both ends is usually larger than that at the middle, resulting in a hard edge and a convex edge, also resulting in uneven dyeing, edge collapse.

The main measures to solve uneven dyeing and selvage collapse are differential yarn guiding. In traditional yarn guiding, a low frequency movement can be attached, which can make the winding position of yarn guiding change slowly and periodically.

Above all, the winding movement can be improved as follows.

(1) The improvement of yarn guiding movement

$$
\theta(t)=\theta_{J}(t)+k_{1} \theta_{0} \sin \frac{\pi}{k_{2} T_{0}} t
$$

Where $k_{1}, k_{2}$ are adjusting coefficients, refer to experience. $\theta_{J}(t)$ is the basic yarn guiding law determined by the equation (3) to (11). The difference of 
time between the go and return stroke is also considered, which can make $T_{b}($ small $\rightarrow$ big $)>T_{b}($ big $\rightarrow$ small $)$.

(2) Changing package build speed $\left(\omega_{g}, \mathrm{rad} / \mathrm{s}\right)$

$$
\omega_{g}=\omega_{g}^{0}\left(1+k_{3} \sin \frac{\pi}{k_{2} T_{0}} t\right)
$$

Where $k_{3}$ is adjusting coefficients of the roller speed, refer to experience, $\omega_{g}^{0}$ is the basic angular velocity of roller $(\mathrm{rad} / \mathrm{s})$.

According to the movement rules established by the above equations, it can realize high speed and precision yarn winding in twisting machine. Similarly, in the multi motor drive system in this paper, the improvement of the yarn guiding movement and the change of friction roller speed can simply realized by changing the control program of yarn guiding movement drive motor and winding rotary movement drive motor. It can be very flexible and accurate.

\section{5 conclusions}

This paper introduces differential equation of yarn winding movement on all kinds of spinning machine. It is the theoretical basis for further research on the various kinds of problem of winding movement. From the angle of mechanics, the faults are analysed about the law of yarn guiding movement of traditional cylindrical cam yarn delivery device and a feasible yarn guiding movement basic law can avoid the inertia impaction at the two re-entry point. The issues of winding tension, winding density, yarn anti-ribboning are also analysed and the method of improving the winding movement is derived. These mathematical models are the theoretical basis of the multi motor driving system of twisting machine.

\section{Acknowledgments}

This work was jointly supported by the key project of the Science and Technology Project of Hubei Educational Committee, China (Q20151603).

\section{References}

1. S. A. Fang. Journal of Donghua University(natural science edition), 21.95 (1995)

2. Z. M. Zhang, S. Q. Mei. W.B.Xiao. Microcomputer Information, 26,21(2010)

3. Z. M. Zhang, S. Q. Mei. Cotton Textile Technology. 34, Oct. 9(2006)

4. F. Xiao, J. S. Mei. Journal of Dandong Textile College. 11, 18(2004)

5. J. P. Wei, L. L.Cai, G.Li, Journal of Textile Research, 28,12(2007)

6. S.Q.Mei, Development and Innovation of Machinery and Electrical Products, 17,2(2004)

7. H.Rong, Cotton Textile Technology.30,5(2002)

8. S. E. Fang, Journal of China Textile University, 21,2(1995)

9. Y. H. Zhou. Journal of Textile Research. 23, 18(2002)

10. J. L. Liu, Gao P. Xiao, Z. X. Zhang. Textile Machinery. 6, 17(2009) 